\title{
Some parameters affecting performance on tandem fixed-interval, fixed-interval schedules
}

\author{
DONALD MELTZER and BETTY JO \\ FREEMAN, Southern Illinois University, \\ Carbondale, Ill. 62901
}

Some temporal parameters of tandem fixed-interval, fixed-interval schedules were investigated. Rats were trained on three tand FI FI schedules. The sum of the initialand the terminal-segment durations was always equal to $2 \mathrm{~min}$, but the ratio of the initial-to-terminal segment duration varied. The distribution of responses in the interreinforcement interval changed as the tandem schedule changed. Latency, defined as the time between the end of the initial segment and the response that initiated the terminal segment, was inversely related to the ratio of initial-segment duration to terminal-segment duration. Response rate was not correlated with this ratio. The distribution of responses during the entire interreinforcement interval was correlated with the ratio.

A tandem fixed-interval, fixed-interval (tand FI, FI) schedule requires that a $S$ complete two successive fixed-interval schedules for reinforcement. A response after the first fixed interval initiates the second fixed interval. At the end of the second fixed interval a response is reinforced and initiates the first interval again. At no time are different exteroceptive stimuli associated with either segment of the schedule.

Since no exteroceptive stimuli are used, a tand FI, FI may produce response patterns similar to those that would be produced by a simple fixed interval. There is no way to decide whether or not such a hypothesis is true because the few studies that describe the results of tandem-interval schedules (e.g., Findley, 1962; Kelleher \& Fry, 1962; Kendall, 1967) used the tandem schedules as control procedures for chained-interval schedules. The description of tandem-schedule results was limited, and both initial- and terminal-segment durations were changed when the results of more than one tandem schedule were reported.

In this experiment, we used three different tand FI, FI schedules in which the sum of the two segment durations was always equal. Any changes in the interreinforcement time on the different schedules therefore could be described as a result of the different schedules. Differences in response rate were also expected as a function of the different schedules.
T3) received 21 sessions of exposure to each of the three schedules in a descending order of initial-to-terminal segment duration ratios, tand FI $96 \mathrm{sec}$ FI $24 \mathrm{sec}$, tand FI $60 \mathrm{sec}$ FI $60 \mathrm{sec}$, tand FI $24 \mathrm{sec}$ FI $96 \mathrm{sec}$. The remaining three Ss (T4, T5, T6) received exposure to the schedules in an ascending order of initial-to-terminal segment duration ratios, tand FI $24 \mathrm{sec}$ FI $96 \mathrm{sec}$, tand FI $60 \mathrm{sec}$ FI $60 \mathrm{sec}$, tand FI $96 \mathrm{sec}$ FI $24 \mathrm{sec}$.

The time between the end of the initial segment and the $S$ 's response that started the terminal segment was recorded and treated as the latency measure. Responses in each FI were also recorded in successive 12 -sec time bins. There were always 10 such time bins during the "entire interreinforcement interval, but different numbers of time bins would be used for recording responses in the initial and terminal FI depending on the tandem schedule. For example, on tand FI $24 \mathrm{sec}$ FI $96 \mathrm{sec}$, the first two time bins were used to record responses during the initial FI, while the last eight time bins we re used to record responses during the terminal FI. When the tand FI $96 \mathrm{sec}$ FI $24 \mathrm{sec}$ schedule was in effect, the first eight time bins were used to record responses during the initial FI, while the last two time bins were used to record responses during the terminal FI. The latency measure therefore was taken at different points in the response distribution. But, since there were no responses during the latency interval, the distortion of the response distribution was minimal.

\section{RESULTS}

The mean total responses per session from the last five sessions on each schedule is shown in Table 1. All six Ss increased their Table 1

Mean Total Responses Per Session Over the Last Five Sessions Exposure to Each Schedule

Schedule of Reinforcement

\begin{tabular}{cccc} 
& tand FI 24 sec & tand FI 60 sec & FI 60 sec \\
\cline { 2 - 4 } Subject & FI 96 sec & 1568.6 & $\begin{array}{c}\text { tand FI 96 sec } \\
\text { FI 24 sec }\end{array}$ \\
\hline T1 & 2064.6 & 668.0 & 1560.8 \\
T2 & 1089.8 & 948.0 & 616.0 \\
T3 & 2596.0 & 530.0 & 825.0 \\
T4 & 469.0 & 1541.0 & 714.0 \\
T5 & 1817.0 & 1004.0 & 2418.0 \\
T6 & 833.0 & 1077.0 \\
\hline
\end{tabular}

Table 2

Mean Number of Reinforcements Obtained Per Hour Over the Last Five Sessions of Exposure to Each Schedule

\begin{tabular}{cccc}
\hline & & Schedule of Reinforcement & \\
\cline { 2 - 4 } Subject & $\begin{array}{c}\text { Tand FI } 24 \mathrm{sec} \\
\text { FI 96 sec }\end{array}$ & $\begin{array}{c}\text { Tand FI } 60 \mathrm{sec} \\
\text { FI } 60 \mathrm{sec}\end{array}$ & $\begin{array}{c}\text { Tand FI } 96 \mathrm{sec} \\
\text { FI 24 sec }\end{array}$ \\
\hline T1 & 20.4 & 20.2 & 24.0 \\
T2 & 20.0 & 21.0 & 23.0 \\
T3 & 20.0 & 21.0 & 22.0 \\
T4 & 18.0 & 21.0 & 25.0 \\
T5 & 21.0 & 22.0 & 26.0 \\
T6 & 20.0 & 24.0 & 24.0 \\
\hline
\end{tabular}



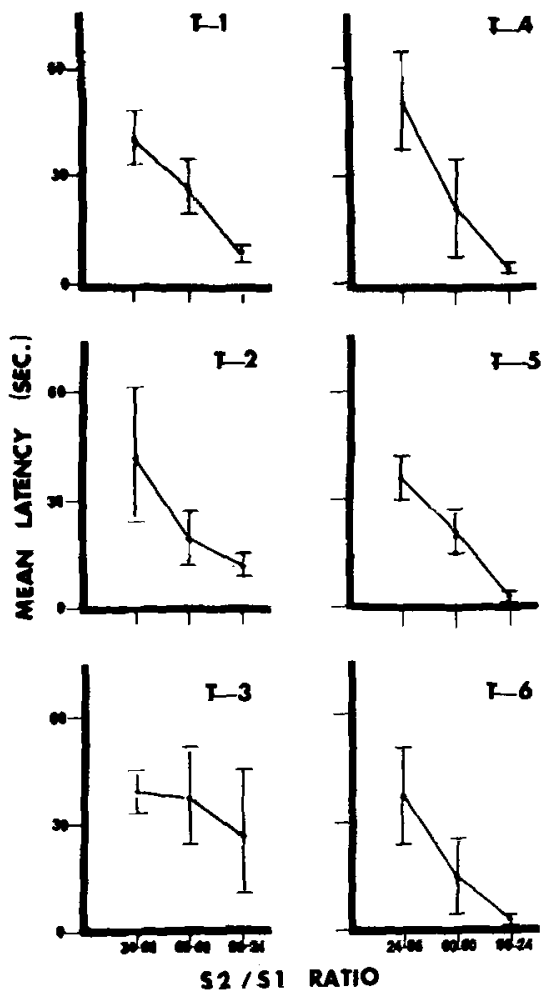

Fig. 1. Mean latency per reinforcement in seconds on each of the three tandem schedules over the last 5 days' exposure to each schedule.

mean number of responses per hour almost every time the ratio of initial-to-terminal segment duration changed, both when it increased and when it decreased. As Table 2 shows, the change in response rate was independent of changes in reinforcement frequency. For three of the Ss (T4,T5, T6), the increase in rate was accompanied by an increase in reinforcement frequency. For the remaining $\mathrm{Ss}(\mathrm{T} 1, \mathrm{~T} 2, \mathrm{~T} 3)$, an increase in rate was accompanied by a decrease in reinforcement frequency.

The fact that reinforcement frequency was closely related to the ratio of initial-to-terminal segment duration is shown by the latency data in Fig. 1. The mean interval between the end of the initial segment and the response that initiated the terminal segment was inversely related to the size of the ratio of initial-to-terminal segment duration. Latency changes were difficult to interpret in this situation. On the one hand, latency changed when the ratio of initjal-to-terminal segment duration changed. On the other hand, latency changes affected the total duration of the

Fig. 2. Mean percentage of total responses in each of the 12-sec time bins over the last five sessions' exposure to each of the three tandem schedules. interreinforcement interval. Changes in the interreinforcement interval, however, might have affected latency independently of changes in the ratio of initial-to-terminal segment duration. Therefore, it is probably best to say that latency was correlated with the ratio of initial-to-terminal segment duation rather than that latency changes were caused by changes in the ratio. Schedule sequence apparently did not affect this measure.

The regularity of the latency data may be considered an unusual result in view of the fact that there was no consistent relationship between total response output and the ratio of initial-to-terminal segment duration. The apparent discrepancy disappeared when the latency period was ignored, and the mean per cent of total responses in each of the 10 successive 12 -sec bins that constituted an interreinforcement interval was calculated. The mean number of responses per bin was calculated and then divided by the number of reinforcements obtained in that session. This value was then divided by the mean number of responses per reinforcement to give the percentage distribution of responses during the interreinforcement interval. The mean of these distributions was calculated for the
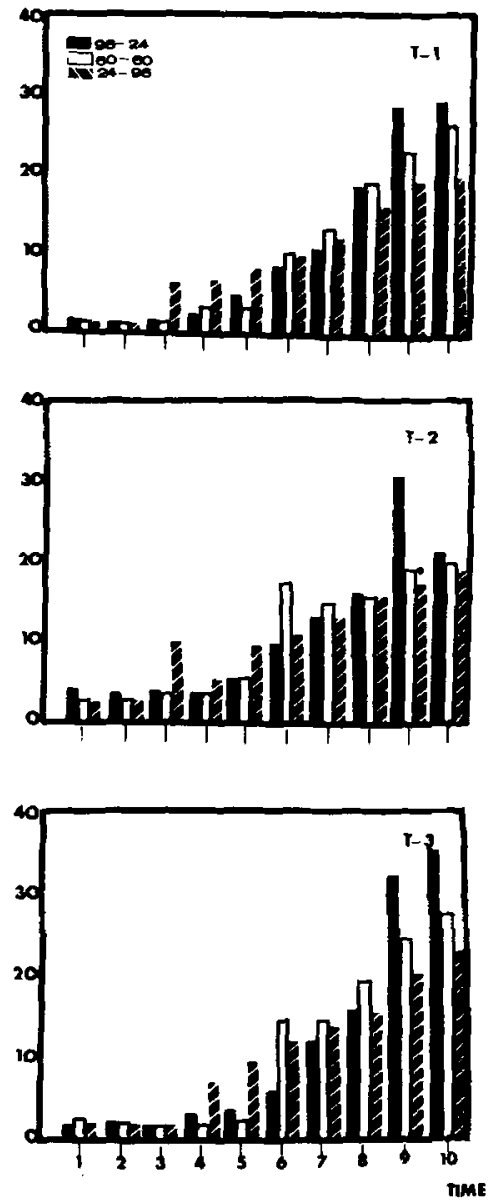

last five sessions on each tandem schedule and is shown in Fig. 2. All six Ss had their flattest response distributions when they were on tand FI $24 \mathrm{sec}$ FI $96 \mathrm{sec}$, and their steepest distributions on tand FI $96 \mathrm{sec}$ FI 24 sec. Apparently, the ratio of initial-to-terminal segment duration had a consistent effect on both response distribution and latency. While increases in response rate might simply have been a result of prolonged exposure to an FI schedule, the changes in response distribution were strong evidence that the tandem schedule was controlling the behavior.

\section{DISCUSSION}

The fact that latency changed as a function of the ratio of the initial-to-terminal segment duration ratio can be explained by an analysis of the prevailing response rate at the time of the transition from the initial to the terminal segment. If response rate was relatively low, latency was longer than if the transition occurred when rate was relatively high. Low response rates were prevalent when the transition came early in the interval and latency was long. High response rates were prevalent when the transition came late in the in terval and latency was short.
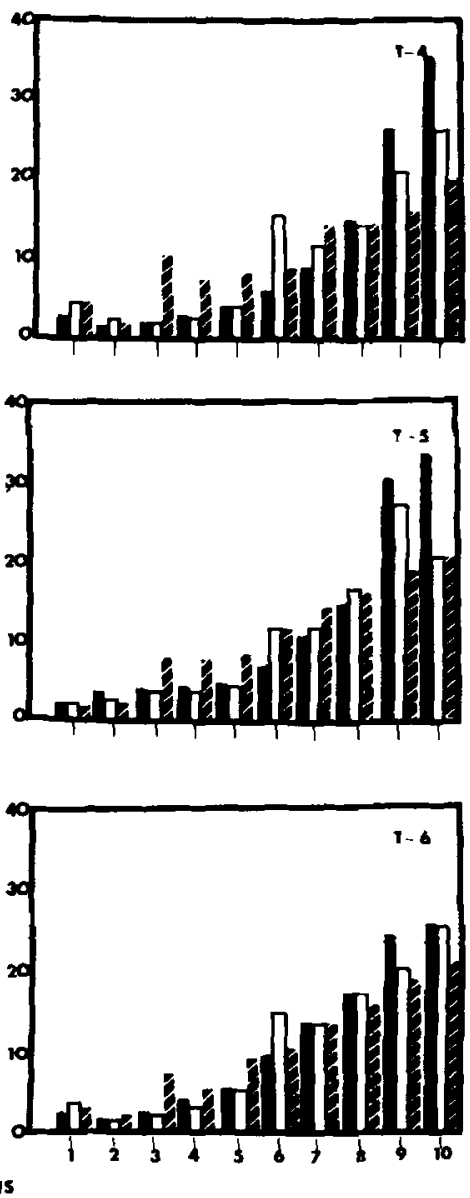
The distribution of responses during the interreinforcement interval was also a function of the ratio of the initial-to-terminal segment duration. This result may be explained by the fact that the different ratios provide differential reinforcement for high rates at different points in the interreinforcement interval. For instance, a tand $24 \mathrm{sec} 96 \mathrm{sec}$ schedule required high rates early in the interreinforcement interval if the $S$ were to maximize the frequency of reinforcement. Even though response rate increased near the end of the interreinforcement interval, the relatively high rates early in the interval would produce a relatively flat response distribution. A tand $96 \mathrm{sec} 24 \mathrm{sec}$ schedule, on the other hand, would provide no differential reinforcement for responding early in the interreinforcement interval. Ss could therefore be expected to show the steeply curved response distribution characteristically observed when a fixed-interval schedule is used.

\section{REFERENCES}

FERSTER, C. B., \& SKINNER, B. F. Schedules of reinforcement. $\mathrm{New}$ York Appleton-Century-Crofts, 1957

FINDLEY, J. D. An experimental outline for building and exploring multi-operant behavio repertoires. Journal of the Experimental Analy sis of Behavior, 1962, 5, 113-166.

KELLEHER, R. T. Chaining and conditioned reinforcement. In W. K. Honig (Ed.), Operant behavior-Areas of research and application. New York: Appleton-Century-Crofts, 1966. P. 160.

KELLEHER, R. T., \& FRY, W. F. Stimulus functions in chained fixed-interval schedules. Journal of the Experimental Analysis of Behavior, 1962, 5, 167.

KENDALL, S. B. Some effects of fixed-interval duration on response rate in a two-component chain schedule. Journal of the Experimental Analysis of Behavior, 1967, 10, 341.

\section{Elicitation of stimulus-bound behavior in guinea pigs ${ }^{1}$}

\begin{abstract}
ANTHONY G. PHILLIPS ${ }^{2}$ and ELLIOTS. VALENSTEIN, Fels Research Institute, Yellow Springs, Ohio 45387
\end{abstract}

The elicitation of stimulus-bound eating, drinking, and gnawing in the guinea pig is described. In contrast to experience with the rat, hypothalamic stimulation elicits eating more frequently than drinking in the guinea pig and the probability of occurrence of stimulus-bound behavior is much higher.

A great number of studies have investigated the consummatory behavior that could be elicited by electrical stimulation of the hypothalamus. This "stimulus-bound" behavior has included, among others, eating, drinking, hoarding, stalking-attack, and sexual behavior (cf. reviews by Valenstein, Cox, \& Kakolewski, 1969). Although the behavior elicited has usually been interpreted as a concomitant of the activation of the neural state underlying such drives as hunger, thirst, and other biological needs, this view has recently been questioned. It has been shown, for example, that animals displaying eating or drinking in response to hypothalamic stimulation differ in significant ways from animals that are known to be hungry or thirsty (Valenstein, Cox, \& Kakolewski, 1968b; Valenstein, Kakolewski, \& Cox, 1968). The hypothesis has been postulated that hypothalamic stimulation does not evoke specific drives, but rather activates the neural substrates of a configuration of responses that are adaptive for a given species. ${ }^{3}$ From this point of view, it becomes important to examine the responses that can be elicited by hypothalamic stimulation in a variety of species. We report here the results of a study of the behavior that was elicited by hypothalamic stimulation in the guinea pig.

\section{SUBJECTS}

The Ss were seven male guinea pigs $(450-500 \mathrm{~g})$ purchased from Hilltop Lab Animals, Inc. (Scottdale, $\mathrm{Pa}$.).

\section{PROCEDURE}

The guinea pigs were implanted with two bipolar electrodes (Valenstein, Hodos, \& Stein, 1961) in the hypothalamus at the left and right of the midline. With the skull leveled in the stereotaxic instrument, the electrodes were inserted $4.0 \mathrm{~mm}$ posterior to bregma, $1.75 \mathrm{~mm}$ lateral and $11.0 \mathrm{~mm}$ below the dorsal surface of the calvarium. The histological information for 10 of the 14 electrode placements is summarized in Fig. 1.

The guinea pigs were tested with the same procedure that has become standardized for the rat in our laboratory (Valenste in, Cox, \& Kakolewski, 1968a). The animals were placed in a Plexiglas chamber containing a water bottle with metal drinking tube, a wooden wedge anchored to the floor, and Purina Lab Chow pellets scattered on the floor. A dish of Purina Guinea Pig Chow was also available. The animals were stimulated for 30-sec periods with a 60-cycle sine wave current, which was gradually raised until the animals clearly responded to the stimulation by moving around the cage in what has been described as a "searching pattern." Following the establishment of an appropriate current level, three standard tests consisting of $2030-\mathrm{sec}$ stimulation periods, each separated by a $60-\sec$ interstimulus period, were administered at each electrode site. An $\mathrm{O}$ scored the occurrence of eating, drinking, and gnawing on the wooden wedge. Eating or drinking were scored only when there was clear evidence that the food or water were ingested. Gnawing consisted of biting off pieces of wood from the wedge.

\section{RESULTS AND DISCUSSION}

The results are summarized in Table 1. Two of the animals (four electrode placements) are excluded as the stimulation only barely aroused the animals and the histological examination revealed that the electrodes had partially penetrated through the ventral surface of the brain. It can be seen from an inspection of the table that the majority of the electrodes elicited eating. Drinking and gnawing were observed in fewer instances. Most animals ate both the rat pellets and the guinea pig chow when 